\section{EWMA 2012}

\section{European Wound Management Association \& AWA}

Termin: 23. bis 25. Mai 2012

Ort: Wien, Austria Center Vienna Themen: Wundheilung - unterschiedliche Perspektiven, ein Ziel. Medikamente in der Wundheilung, Stellenwert des Debridements bei akuten und chronischen Wunden, spezielle Wunden, Onkologie und Wunden, Wundheilung jenseits von Verbänden, Unterdrucktherapie, Schulung und Diabetischer Fuß.

Informationen: EWMA Secretariat, Nordre Fasanvej 113, 2, DK-2000 Frederiksberg, Tel.: +45/70/20 03 05, Fax: +45/70/20 03 15, E-Mail: ewma@ewma. org; AWA Secretariat, P.O. Box 6, 1106 Wien, Tel: 01/879 03 79, Fax: 01/879 03 79-90, E-Mail: office@a-w-a.at Internet: www.a-w-a.at

\section{KARDIOLOGIE}

\section{Jahrestagung der ÖKG 2012}

Termin: 30. Mai bis 2. Juni 2012

\section{Ort: Salzburg Congress} Themen: Unter anderem: Echokardiographie in der Praxis, Lungenembolie: Standortbestimmung, Herausforderungen bei

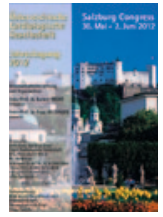
kongenitalen Aortenerkrankungen, Medikamentöse Prävention beim Herzgesunden mit Risikofaktoren, der Notfall im Herzkatheterlabor, Herzinsuffizienz - eine interdisziplinäre Herausforderung, Komplette vs. Inkomplette Revaskularisation: Alles (UN) klar nach STICH?? Antithrombotische Strategien: Effektivität versus Sicherheit. Informationen: Edith Tanzl, Ingrid Lackinger, Universitätsklinik für Innere Medizin II, Abteilung für Kardiologie, Währinger Gürtel 18-20, 1090 Wien Tel.: 01/40400-4614, Fax: 01/40400-4216, E-Mail: office@atcardio.at

\section{$4^{\text {th }}$ UPDATE}

\section{Endoscopic Skills 2012}

Termin: 1. Juni 2012

Ort: Paracelsus Medizinische Universität Salzburg (PMU), Hörsaal 1, Hansjörg Wyss Haus, Strubergasse 21, 5020 Salzburg, Österreich
Thema: Update on highest-level endoscopic analysis and skills for en-bloc resection of early mucosal neoplasias. Tips for high-definition diagnostic endoscopy, the tricks and hurdles of endoscopic en-bloc resection, and diagnostic procedures to support curative strategies for Gl tumors such as endoscopic ultrasound and enteroscopy. Veranstalter: Frieder Berr (AT), Helmut Messmann (GE), Thierry Ponchon (FR), Hans Seifert (GE)

Informationen: Dr. Tobias Kiesslich, UK Innere Medizin I, PMU/SALK, Müllner Hauptstrasse 48, 5020 Salzburg E-mail: endo12@pmu.ac.at Internet: www.salk.at/m1.html

\section{ÖGIAI}

\section{Gemeinsame Jahrestagung}

der Deutschen

Gesell. für Internistische Intensivmedizin und Notfallmedizin und der Österreichischen Gesellschaft für Internistische und Allgemeine Intensivmedizin Termin 6. bis 9. Juni

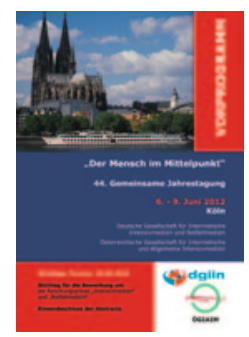
2012

Themen: Der Mensch im Mittelpnkt: Ethik, Struktur und Qualität der Intensivmedizin, Risiko- und Fehlermanagement, Kostenfragen, Kommunikation, Angehörige, Umsetzung der Leitlinien , Sepsis, Kardiogener Schock, Sedierung, Reanimation, Pro-Kontra Sitzungen zu umstrittenen Themen, Hygiene auf der Intensivstation, Nierenersatztherapie, Herzrhythmusstörungen, Antikoagulation, Der alte Intensivpatient, Neurologische Probleme, Nosokomiale Infektionen, ARDS und ALI,

Gastroenterologische Probleme, Akutes Leberversagen, Hepatorenales Syndrom, Akute Pankreatitis, Gastrointestinale Blutung, Bildgebung in der Intensivmedizin, Notfallaufnahme: die ersten 24 Stunden, SOP's in der Intensivmedizin, Organversagen - Organersatztherapie, Intoxikation, Infektiologie, Berufliche Perspektiven in der Intensivmedizin. Informationen: Tagungssekretär, Dr. Andreas Niedeggen, St. Antonius Hospital, Innere Medizin, Dechant-DeckersStr. 8, D - 52249 Eschweiler,

Tel.: +49/2403/761784

Fax: +49/2403/ 761827, E-Mail: andreas. niedeggen@sah-eschweiler.de

\section{ÖGC}

\section{3. Österreichischer Chirurgenkongress}

Termin: 7. bis 9. Juni 2012

Ort: Salzburg

Hauptthemen: Endoskopie und Interventionen, Radiologie und Interventionen, Endokrine Chirurgie, Herzchirurgie, Thoraxchirurgie, Mammachirurgie, Leber-Pankreaschirurgie, Gastrointestinale und Kolorektale Chirurgie, Metastasenchirurgie, Qualitätsmanagement in der Chirurgie, Stellenwert der Chirurgie in der Onkologie - multimodale Strategien, Proktologische Chirurgie, Qualitätssicherung in der Hernienchirurgie (Hernia Med), Minimal Invasive Chirurgie, Adipositaschirurgie, Refluxchirurgie, Komplikationsmanagement, Aus- und Weiterbildung, Simulation, Perioperative Ernährung, Perioperative Schmerztherapie, Young Surgeons Forum, Innovationen und Zukunftsperspektiven, Entzündliche Darmerkrankungen, Akutes Abdomen, Palliativchirurgie

Informationen: Fortbildungsakademie der Österreichischen Gesellschaft für Chirurgie, Univ. Prof. Dr. Hans Werner Waclawiczek, Univ.-Klinik für Chirurgie, Private Medizinische Universität Salzburg (PMU), Müllner Hauptstraße 48, 5020 Salzburg, E-Mail: h.w.waclawiczek@salk.at Tel.: 0662/4482-51100

Mobil: 0664/3217332

Fax: 0662/4482-51108

\section{IFIMP 2012}

\section{Innsbrucker Forum für} Intensivmedizin und Pflege

Termin: 13. bis 14. Juni 2012

Ort: Innsbruck Congress

Thema: "Evidence meets Eminence“ - ein gutes Rezept macht noch keinen guten Koch. Unter anderem Ethische Betrachtungen zu Leitlinien, Hämotherapie-Richtlinie der ÖGARI für die Intensivmedizin, Evidenzbasierte Beatmungskonzepte, Albumin in der Intensivmedizin , Evidenz in der Therapie des Leberversagens.

Informationen: Univ.-Klinik für Allgemeine und Chirurgische Intensivmedizin, Anichstraße 35, 6020 Innsbruck E-Mail: congress@ifimp.at Internet: www.intensiv-innsbruck.at 